\title{
Author Index Vol. 64, 1997
}

Abe,M. 206 Adapinar, B. 236 Aizawa, H. 59 Aksu,H.S.Z. 73 Alfaro,V. 118 Aliani,M. 111 Allegra,L. 397,416,445 Altin,S. 162 Alvarez, R. 220 Ambrosino, N. 263 Ariaansz, M. 29 Arnulf,I. 159 Arslan, R. 236 Atzori, L. 145

Bacci,E. 285 Bancalari, L. 285 Banerjee, A.K. 381 Bannenberg, G. 145 Baños, V. 220 Barlow, C.W. 131 Bärtsch,P. 436 Basnyat, B. 445 Bauer, C. 300 Baur,X. 35 Beitzke,A. 300 Benden,C. 211 Berg, J.W.K. van den 114 Biasin,C. 371 Birrer,P. 342 Bisetti,A. 165 Boehler,A. 375 Boillot,O. 173 Bolliger, C.T. 388 Boss,J.H. 384 Braun,J. 350 Breceda,V. 358 Bressollette, L. 326 Brusselle,G. 102 Burioka,N. 268

Canteras, M. 220 Carino,M. 111 Carnevali,S. 285 Casale,R. 251 Casaulta Aebischer, C. 342 Catalini,G. 367 Cecarini, L. 367 Chan,Ch.H.S. 152,224 Chan,T.-H. 224 Chang, M.-C. 319 Chaouat,A. 187

Charpentier, C. 187 Chen,C.-Y. 319 Chen,G.J. 358 Chiang, C.-D. 319 Chinet,T. 121 Chiyotani, A. 176 Cianchetti, S. 285 Çikrikçioglu, S. 162 Clavier, J. 326 Clini,E. 138,263 Cogo,A. 397,416,445 Congiu, L. 145 Constantopoulos, S.H. 81 Cordier,J.F. 173 Cornelissen, P.J.G. 273 Corriga, A.M. 145 Cramer, S.G. 96 Cuesta,C. 244

Dalhoff,K. 350 Dalquen,P. 182 Darriba,J. 244 Davey,P.P. 131 De Diego, A. 304 DeNitto,P. 50 Demircin, M. 364 Dente,F.L. 285 Derenne, J.-P. 159 Di Franco, A. 285 Dinesh, S.V.R. 358 Dogan,Ü.B. 73 Domingo, C. 240 Dorow, P. 2 Dür,P. 200 Duran,W. 66 Eber,E. 300 Ebihara, S. 76 Ehrhart,M. 187 Elad,S. 170 Erginel, S. 236 Ewig,S. 194

Fehr,B. 152 Ferencic, Z. 247 Ferrari, M. 371 Fianchini, A. 367 Fickers, M. 114 Firat,P. 364 Flintrop, M. 66 Fogue, L. 244 Fruhmann, G. 10

Fujimura, S. 54 Fujiwara, H. 336 Fukuyama, Y. 316 Furuno, T. 206

Gaeng,D. 233 Garcia, A. 220 Garcia-Pachon, E. 392 García-Río, F. 229 Garcia Rull, S. 244

Garma, L. 159 Gaudó,J. 296 Giannini, D. 285 Gibbs,A.R. 236 Gille,D. 173 Gillissen,A. 16,23

Giuntini,C. 50,285 Gomez, J. 220 Gotoh,K. 336 Grädel,E. 182 Graf Bigler, U. 342 Grbac,II. 247 Greefhorst, L.P.M. 273 Guidi,L. 165

Hagimoto,N. 291 Hamatake, M. 316 Hara,N. 59,206,291 Harmanci, E. 236 Hasper,E. 194 Hata,J. 307 Hayashi,T. 310 Hayashi,Y. 108 Hays, A.M. 358 Herzog, H. 1 Hida,W. 76 Hidaka,K. 206 Hirakawa, S. 336 Ho, A. 224 Hoffmann, U. 200 Hoheisel,G 152 Höllinger,P. 233 Homeyer,P. 159 Hoshino, E. 268 Houston, C.S. 398 Hsu,J.-Y. 319 Hsu,W.-H. 319 Huchon, G. 121

Ibáñez,J. 118 Ikebata,Y. 179 Ioli,F. 111

Ishida,T. 316 I§iksoy, S. 236 Iwabuchi, S. 54 Izbicki,G. 388

Jaworska, M. 16 Jimenez, D. 296 Joos, G. 102

Kakkar,N. 381 Kamio,K. 108 Kanazawa, M. 307 Karakostas, K.X. 81 Karrazi,R. 371 Kars,A. 364 Katoh,H. 108 Kawai,C. 307 Kawajiri, T. 310 Keistinen, T. 281 Kessler,R. 187 Kido,M. 310,331 Kikuchi,Y. 76 Kivelä,S.-L. 281 Knezevic, F. 247 Koike, K. 54 Kondo,T. 108 Konno,K 176 Ko§ar, F. 162 Koto,H. 59 Kraemer, R. 342 Krieger,J. 187 Kumar, L. 381 Kunitake,R. 291 Künzli,A. 182 Kurosawa, H. 76 Kuwano,K. 291 
Lai,Ch.KW. 224 Lampronti, G. 371 Lantz,R,C. 358 Lastra, E. 296 Lavietes, M.H. 66 LeMoigne,E. 326 Legnani, D. 416,445 Leroyer, C. 326 Leung, R. 224 Licitra, C. 111 Lin,C.-C. 257 Lin,C.-Y. 257 Lin,W.H. 96 Lohri,A. 388 Lorenzo, M. 220

\section{KAHGEIV}

E-Mail karger@karger.ch Fax+416130612 34 http://www. karger. ch

(C) 1997 S.KargerAG, Basel

451

Lüderitz,B. 194 Luis, D.A. de 244 Lundberg, J.M. 145

Maesen, F.P.V. 273 Majori, M. 45 Manganelli, D. 50 Maniati, M.A. 81 Marangoni, S. 138

Marchesani, F. 367 Marczynski, B. 35 Marek,W. 35,211 Marín,A. 240 Marini,C. 50 Mariotta, S. 165 Maruyama, R. 316 Mas, A. 240 Matsumoto,K. 59,313 Mattia,P. 165 Mazzantini, D. 50

Meduri,G.U. 371 Mehnert, A. 350 Melej,R. 45 Mensing, T. 35 Metintas, M. 236 Midorikawa, J. 76 Mirapeix, R.M. 240 Misselevitch, I. 384 Mitsudomi, T. 316 Mitsuyama, T. 206 Mizusawa, A. 76 Modi, M.R. 66 Moldèus,P. 145 Morgül, M. 162 Mottier, D. 326 Moutsopoulos, H.H. 81 Murakami, K. 313

Naeije, R. 429 Nagai,A. 176 Nagashima, K. 336 Nagata,N. 310,331 Nagatomo, H. 331

Nagler,A. 170 Nakamura,Y. 179 Nakanishi,K. 310 Naparstek,E. 170 Nauffal,D. 304

Nauta,J.J.P. 29 Navío, P. 296 Nikaido,Y. 310,331 Nishioka,K. 316 Nomoto,Y. 291 Nomura, M. 336 Noord, J.A. van 114

Odeh,M. 384 Ogawa, H. 76

Oger,E. 326 Ohta,Y. 108 Okabe,S. 76 Oliven,A. 384 Olivieri, M. 371 Ono, S. 54 Or,R. 170

Özdemir,N. 236 Özyurt,H. 162

Padilla-Navas, I. 392 Paggiaro, P.L. 285 Palacios,L. 118 Palla,A. 50 Pallone,G. 165 Pandit, J.J. 86 Papiris, S.A. 81 Pasaoglu, Ö. 236 Pasqualetti, P. 251 Paterson, D.J. 131 Pauwels,R. 102 Pazzagli, M. 50 Pedicelli,G. 165 Pejsa,V. 247 Pela,R. 367 Pérez-Rodríguez, E. 296 Perpiñá, M. 304 Perruchoud, A.P. 152,388 Pesci, A. 45 Philippou, S. 211 Philit,F. 173 Piccoli,M.L. 45 Pileggi,V. 45 Pino, J.M. 229 Pinson,P. 102 Pomey,M.-P. 326 Porta, R. 263 Postmus,P.E. 29 Prados, C. 229 Prieto,M. 304

Qayyum,M.S. 131 Quadri,A. 138 Quadri,F. 388

Racionero, M.A. 229 Ratjen,F. 350 Resch,B. 300 Rider,E.D. 358 Riedel,F. 35,211 Ritter,A.B. 66 Robbins,P.A. 86,131 Robledo,R.F. 358 Roche, N. 121 Rogado,M.C. 304 Rossi, G. 50 Roth,M. 152 Ruacan, §. 364

Ruff,P.W. 152 Ruiz,J. 220 Russi,E. 200 Russi,E.W. 375 Ryrfeldt,A. 145

Sabbatini,A. 367 Saito,S. 268 Saitoh, G. 316 Samaja,M. 422 San Miguel, P. 244 Sanguinetti, CM. 367 Sañudo,J.R. 240 Sarno,N. 111 Sasaki, M. 179 Sasaki, T. 268 Sato,K. 313 Sawa, T. 336 Schäfer,H. 194 Schärling,B. 16 Schechter,D. 170 Schena,M. 138 Schinkewitch, P. 187 Schläpfer,R. 182 Schoene,R.B. 407 Schultze-Werninghaus, G. 1 (

23 Schwaiblmair, M. 10 Serrano,S. 229 Shigyo,M. 59 Shimazu,M. 179 Shimizu,J. 179 Shirato,K. 76 Shishido,H. 313 Singh, S. 381 Smeets, J.J. 273 Soejima, K. 307 Soler,M. 388 Speich,R. 375 Stancic,V. 247 Steurer,J. 200 Streckert, H.-J. 211 Sugimachi, K. 316 Sugio, K. 316 Suyama, H. 268

Taguchi, O. 76 Takano,K. 268 Takata,A. 307 Takata,S. 59 Takeuchi,K. 179 Tamaoki, J. 176 Tamminga, J.J. 29 Tanaka, T. 206 Tanigaki,T. 108 Tanita,T. 54 Tasaka, S. 307 Tekuzman, G. 364 
Thalhofer, S. 2 Theunissen, P. 114 Thrall, R.S. 96 Tollinger,B.J. 358 Torikata,C. 307 Torras, R. 118 Torrelli,L. 165 Torremorell, M.D. 240 Tsakiris, D.A. 152 Tsuda,M. 291 Turcotte, J.G. 96 Tuuponen, T. 281

Urano,T. 108

Vagaggini, B. 285 Valdés,M. 220 Valiño,J. 296 Van Keimpema, A.R.J. 29 Vasishta, R.K. 381 Vetter,W. 200 Vilkman,S. 281 Villamor,J. 229 Viso,N. 35 Vitacca,M. 138,263

Vlachoyiannopoulos, P.G. 81 Vogelmeier, C. 10 Vogt,P. 182

Wald,F.D.M. 273 Wang,S. 358 Watanabe,K. 313 Watanabe,S. 179 Watripont,P. 102 Weder,M. 342 Weder,W. 375 Weitzenblum, E. 187 Whitelaw, W.A. 159 Wickenburg, D. 23

Wiesendanger, T. 173 Wiessmann, K.J. 350 Witten, M.L. 358

Yagi,Y. 336 Yalçm, S. 364 Yamaguchi, K. 307 Yasuda,N. 336 Yoshii,C. 331

Zach,M. 300 Zenklusen, H.R. 182 Zuccali,V. 371 Zueger,O. 388 Zwoll,D. van 16,23

452

Respiration Vol. 64, 1997

Author Index 